\title{
Postures et activité du sujet en formation : de l'intention au geste professionnel
}

Geneviève Lameul

\section{OpenEdition}

\section{Journals}

Édition électronique

URL : http://journals.openedition.org/ripes/1160

DOI : 10.4000/ripes. 1160

ISSN : 2076-8427

Éditeur

Association internationale de pédagogie universitaire

Référence électronique

Geneviève Lameul, «Postures et activité du sujet en formation

de l'intention au geste professionnel », Revue internationale de pédagogie de l'enseignement supérieur [En ligne], 32(3) | 2016, mis en ligne le 20 décembre 2016, consulté le 23 septembre 2020. URL : http:// journals.openedition.org/ripes/1160; DOI : https://doi.org/10.4000/ripes.1160

Ce document a été généré automatiquement le 23 septembre 2020.

Article L.111-1 du Code de la propriété intellectuelle. 


\title{
Postures et activité du sujet en formation : de l'intention au geste professionnel
}

\author{
Geneviève Lameul
}

1 Force est de constater que depuis quelques années, tant dans le champ de la recherche que dans celui de la pratique, le terme "posture » est de plus en plus employé. Cette recrudescence d'usage dans le langage académique et dans celui de la pratique, d'un mot appartenant initialement aux secteurs médical et sportif, doit d'autant plus questionner praticiens et chercheurs que ce dernier n'est que très rarement défini. Plusieurs raisons de cette montée en puissance de l'usage du mot dans le champ des sciences humaines peuvent être évoquées :

- le développement de l'usage du numérique relève d'une révolution culturelle qui met en cause les structures et l'organisation de l'action humaine et le rapport à la réalité ;

- le mot "posture » s'impose au plan épistémologique et praxéologique en réaction à la dématérialisation du monde comme une façon de rappeler l'importance de la dimension humaine ;

- la préoccupation de la posture s'inscrit dans un mouvement sociétal global qui fait une plus large place au corps, à l'émotion, au sentiment ;

- la complexité des mondes politiques, économiques et sociaux liée à la mondialisation est si perturbante et parfois éprouvante que les humains ont besoin de retrouver et de se recentrer sur leurs racines : écouter les sons et les images qui ont façonné leur enfance pour sentir et comprendre tout ce qui les constitue de manière symbolique et sensible.

2 C'est sur la base de cet ensemble de constats et d'interrogations qu'est né le projet de ce numéro spécial, intitulé Postures et activité du sujet en formation: de l'intention au geste professionnel, qui donne la parole à des auteurs qui interpelés par ces phénomènes, ont commencé à travailler sur ou autour de cette notion. S'appuyant sur quelques définitions du terme " posture » existantes en sciences humaines et sociales, ce numéro thématique se donne pour objectif de les mettre en perspective de notions afférentes, de les discuter, de les enrichir, et de les questionner dans une visée de 
conceptualisation. La présentation de son usage en différents contextes d'éducation donne un aperçu de son potentiel et souligne plusieurs dimensions convergentes. $\mathrm{Ce}$ numéro vise également à faire un premier point sur la manière d'opérationnaliser cette notion complexe en proposant des grilles d'analyse utilisables dans les champs de pratiques éducatives, tout particulièrement en pédagogie universitaire.

\section{1. Éléments de définition de la notion de posture}

Dans le dictionnaire des concepts de la professionnalisation, la posture professionnelle est désignée par Mulin (2014) comme « la situation dans laquelle agit un professionnel, tout autant que le système d'attitudes qu'il adopte dans cet exercice » (p. 213). Dans un sens générique, la posture est considérée comme un état mental façonné par les croyances, les valeurs et les intentions qui donnent sens aux actions d'une personne (Lameul, 2006, 2008). Empreinte de toute la dimension interne et intime du sujet, la posture assure ce passage d'une intériorité à une extériorité qui s'exprime dans et par le geste professionnel. Sont alors en question les modalités de la traduction qui s'opèrent entre des mondes de nature et de niveaux différents de même que les modes de passage de l'invisible au visible.

Pour en approcher la conceptualisation, il paraît nécessaire dans un premier temps de situer la notion de posture par rapport à des notions en proximité (attitudes, conduites, style, genre, ethos, habitus). Notre quête d'une définition conceptuelle de la notion de posture conduit à souligner la mise en équivalence régulière des notions de posture et d'attitude, dans le langage commun. Le nouveau petit Robert de la langue française donne de la notion de posture, la définition suivante « position, attitude particulière du corps, pose " (Posture, 2010, p. 1978) tandis qu'il affiche pour attitude "manière de tenir son corps, position qu'on lui donne, posture » (Attitude, 2010, p. 173) en spécifiant qu'en psychologie sociale, la notion d'attitude correspond à « une disposition profonde durable et d'intensité variable à produire un comportement donné » (Attitude, 2010, p. 174). Notre exploration scientifique et empirique continue depuis 2006 nous conduit à souligner que dans plusieurs citations, les termes de posture et d'attitude coexistent, ce qui nous laisse penser que cette juxtaposition traduit un souhait de souligner des nuances de sens. Par exemple, une citation de Trocmé-Fabre (1999) reprise par Carré (2005) a été déterminante dans le choix que nous avons fait de nous engager dans cette tentative de définition distinctive de la notion de posture: "L'actualisation de l'apprenance [qui] dépend donc de la posture, du positionnement, de l'attitude, de l'intention, des conditions dans lesquelles se trouve l'organisme apprenant et de la façon dont il est relié à son environnement, aux autres et à lui-même » (p. 192).

Correspondant aux réactions d'un individu par rapport à des objets, des idées ou des personnes, les attitudes comportent selon Vallerand (1994), plusieurs facettes :

- une facette mentale : la représentation de l'objet, de l'idée ou de la pensée considérée ;

- une facette affective: les émotions (représentation, rejet, mépris, tendresse, amour...) suscitées à leur évocation ;

- une facette comportementale : les conduites d'évitement ou de rapprochement adoptées à leur égard.

6 L'attitude n'est donc pas simplement une opinion, un ensemble pur de représentations, c'est aussi un ensemble d'affects, de réactions émotives qui contribue à guider nos actions et engage l'individu au-delà des seules idées et représentations. L'attitude est 
un état à la fois « mental et neural » note Allport (1954, p. 43), soulignant par là, sa forte inscription émotive et corporelle.

7 La notion de posture est également dans une certaine proximité de celle d'habitus, tel que défini par Bourdieu (1987) en tant que système de dispositions durables intériorisées par les individus du fait de leurs conditions objectives d'existence, et qui fonctionne comme principes (schèmes) inconscients d'action, de perception et de réflexion. Deux composantes de l'habitus peuvent être distinguées :

- l'éthos désigne les principes ou les valeurs à l'état pratique, la forme intériorisée et non consciente de la morale qui règle la conduite quotidienne;

- l'hexis corporelle correspond aux postures, dispositions du corps, rapports au corps, intériorisés inconsciemment par l'individu au cours de son histoire.

Produit de la position et de la trajectoire sociale des individus, c'est un mécanisme d'intériorisation de l'extériorité qui fait que des sujets situés dans des conditions sociales différentes, vont acquérir des dispositions différentes. Produit de l'expérience passée et présente, l'habitus est une structure interne toujours en voie de restructuration et en même temps présentant une forte inertie. A l'interface interneexterne, la notion de posture renvoie donc à celle d'habitus et appelle une clarification dans le positionnement de ces deux notions. Se pose la question de savoir si la posture est incluse dans l'habitus et/ou si elle représente ce qui fait relation entre éthos et hexis. L'habitus est ce que l'on a acquis, mais qui s'est incarné de façon durable dans le corps sous forme de dispositions permanentes. Il est le produit de conditionnement qui tend à reproduire la logique objective des conditionnements mais en lui faisant subir une transformation. Dans la mesure où il y a réunion de deux faces, l'une objective (structure), l'autre subjective (perception, évaluation), il peut être dit que l'habitus intériorise l'extérieur et qu'à l'inverse, il extériorise ce qui vient de l'intérieur. Et c'est dans ce mouvement interne-externe que la notion de posture lui ressemble et qu'il peut être dit que la posture est au service de l'habitus.

\section{Contribution des auteurs de ce premier numéro}

Considérant que la posture professionnelle désigne aussi en partie la situation dans laquelle se fait l'action, plusieurs situations de tensions au sein desquelles se construisent/se consolident/se déploient les postures professionnelles sont abordées et discutées en différents contextes. Chacun des cinq textes qui composent ce numéro apporte sa contribution spécifique pour une meilleure compréhension de la construction d'une posture professionnelle.

Anne Jorro s'intéresse à un public particulièrement interpelé par ces questionnements autour de la notion de posture parce que l'évolution de son métier l'y oblige, celle des conseillers en évolution professionnelle. Dans le cadre d'une recherche formation, elle montre comment le conseiller est en situation de tension entre une mobilisation des outils habituels du conseil et une ouverture d'esprit qui dans la relation à l'autre va générer des configurations qui vont permettre à ce dernier de saisir des possibilités d'action et d'évolution professionnelle. La posture implique un travail sur soi qui dans une démarche analytique, croise les intentions et les actes. L'analyse des situations de conseil permet d'identifier des manières d'agir auprès d'autrui qui renvoient à trois postures : accueil, écoute et médiation. 
11 A partir de la reprise de trois de ses terrains de recherche, Sylvain Starck tente de comprendre les dynamiques liées à l'adoption d'une posture dans l'exercice professionnel. Il suit tout particulièrement les acteurs dans leur engagement professionnel en étant attentif à la manière dont ils se rapportent aux situations, aux autres et à eux-mêmes. En se référant aux travaux de Clot (2007), il parle de posture de métier qui autoriserait une extension du « corps propre »; la posture rendant compte d'un rapport simultané aux situations, aux autres et à soi-même crée une tension qu'il s'agit de rendre cohérente en pratique. Sylvain Starck fait plusieurs propositions pour travailler la posture en formation: elle est un analyseur pour étudier le processus intégratif lié à l'activité; la posture se rapproche du style de métier et nécessite d'accompagner les professionnels dans leur capacité à composer entre dimensions instituées du métier et appropriation singulière, à incorporer une posture. En tant qu'intégrateur de l'agir professionnel, il le voit comme un trait d'union entre corps propre et corps social.

12 Jean Yves Robin et Nathanael Wallenhorst s'intéressent à une question encore peu travaillée à l'université, celle de la réorientation des étudiants. C'est dans cette situation pédagogique spécifique inhabituelle et inconfortable qu'ils perçoivent une interpellation intéressante de l'enseignant-chercheur, dans sa position académique. Ils relatent une expérience d'une vingtaine d'année dans un dispositif aux frontières de l'université qui met en lumière toute l'importance d'une plus grande prise en compte des dimensions liées au sensible, à l'existentiel, au vocationnel. Face à ces étudiants en voie de réorientation, c'est d'une réelle mise en question de la place du savoir existentiel à l'université à côté des savoirs et des savoir-faire dont il est ici question. L'enseignant-chercheur est particulièrement interpelé dans sa posture en entrant dans ce champ éducatif auquel il n'est pas préparé.

13 Christelle Lison et Denis Bédard se questionnent sur les postures d'enseignants-tuteurs mettant en œuvre la méthode d'apprentissage par problèmes dans deux disciplines : la médecine et le génie. Par le biais d'entrevues semi-dirigées, d'observations et d'entretiens d'auto-confrontation auprès de 13 enseignants-tuteurs, ils font le constat que ceux de médecine et de génie n'adoptent pas la même posture épistémique. Parmi les propositions d'explication formulées : la manière dont l'APP a été mise en œuvre dans chacun des programmes; le profil du groupe d'étudiants, la nature de la situationproblème. Plusieurs de leurs constats retiennent notre attention, car ils entrent en résonnance avec les résultats de la recherche $H y$ Sup $^{1}$ évoqués dans l'article de Nathalie Deschryver et Geneviève Lameul : le discours des tuteurs est, la majorité du temps, cohérent avec leurs actions; ils sont généralement conscients de la posture qu'ils adoptent; il y a cohérence entre la posture et le style d'animation qu'ils mettent en place lors des rencontres d'APP.

14 Enfin, Nathalie Deschryver et Geneviève Lameul reprennent une partie des travaux de la recherche Hy Sup pour approfondir une réflexion sur les moyens méthodologiques mobilisés en recherche afin d'étudier les postures professionnelles, dans la perspective d'en proposer une grille de lecture pour l'action. Comme l'illustrent les articles précédents, la notion est si complexe et subtile qu'elle appelle un questionnement en profondeur et en extension (prise en compte de multiples dimensions spécifiques à chaque contexte). L'étude plus particulière de deux outils en montre les avantages et les limites et souligne la difficulté à embrasser l'ensemble des dimensions d'une posture. Il y est souligné la nécessité d'explorer en contexte les dimensions 
biographiques et socio-affectives qui participent à construire une posture professionnelle. Une méthode mixte associant questionnaire et entretien est probablement la plus à même de rendre compte de l'alignement qui s'opère entre intentions, croyances et actions, à un moment donné pour un sujet dans le vécu d'une situation particulière.

\section{Apport spécifique de ce numéro}

Du point de vue de la recherche, la contribution à la conceptualisation de la notion de posture qu'apporte ce numéro spécial peut être mise en relation avec la réflexion amorcée par Albero et Guérin (2014) dont la note de synthèse fait un bilan des travaux centrés sur la notion d'activité en sciences de l'éducation. Reprenant des travaux de Linard $(1989,2002)$, ils préconisent de faire de l'analyse de l'activité un cadre organisateur pour construire une référence commune aux sciences qui étudient l'activité humaine. Des trois types de contraintes pesant sur l'activité que mettent en évidence Albero et Guérin (2014) dans l'étude de plusieurs courants de recherche relatifs à l'activité humaine, sont ici plus spécifiquement abordées les contraintes liées à l'état du sujet (capacités, compétences, connaissance, motivation et état du moment) dans leur articulation avec ceux d'ordre culturel (valeurs, normes, artefacts matériel et symbolique) et en lien avec ceux qui relèvent de la tâche. Restent à affiner des grilles d'analyse susceptibles d'objectiver la subjectivité, de prendre en compte les processus socio-historiques des sujets et d'appréhender les espaces-temps physiques et symboliques qui les situent de manière concomitante avec les registres biologique, psychologique et social. La notion de posture peut alors être vue comme l'un des objets d'étude de ce vaste programme de recherche pluridisciplinaire autour de l'activité du sujet en situation (travail, formation, apprentissage) qu'ils proposent. A la fois, elle sert à spécifier l'action incarnée et à caractériser l'acteur qui l'assume ; à la fois, elle traduit le lien qui se fait entre le contexte/l'environnement et la personne qui effectue un geste professionnel. Focalisé sur l'alignement entre croyances, valeurs et actions, la posture d'un sujet traduirait la mise en cohérence et l'équilibre entre ses environnements interne et externe, ses dimensions socio-historiques, ses caractéristiques rationnelles et intuitives, cognitives et sensibles, etc.

Enfin, en cohérence avec les lignes directrices de la Revue internationale de pédagogie de l'enseignement supérieur, l'interrogation de la notion de posture se fait dans la perspective d'être utilisable dans le champ de pratiques de la formation des adultes et tout particulièrement dans celui de la pédagogie universitaire. Parce que nous sommes attachée à l'articulation des savoirs et des pratiques (Lameul \& Loisy, 20014), ce numéro a la préoccupation de montrer en quoi et comment ces réflexions et ces outils développés pour appréhender ce qui relève de la posture chez un professionnel, peuvent être exploités avec et par les praticiens. Loin de se réduire aux seules dimensions cognitives, les apprentissages mettent en jeu la totalité du sujet dans sa corporéité, ses émotions, ses perceptions sensibles (intuition, kinesthésie, créativité, sensibilité, etc.). Les conditions à réunir pour que ce travail puisse se faire, sont analysées en plusieurs contextes: comment s'articule l'incorporation d'un passé et d'expériences socialisatrices antérieures avec le moment présent à vivre immédiatement? Quelles conditions de confort réunir pour que les tensions générées se vivent positivement et participent à l'émancipation des personnes? Comment 
repérer la combinaison de ce qui est de l'ordre de l'histoire personnelle du sujet et de ce qui relève de son collectif d'appartenance?

\section{Invitation à poursuivre}

17 Dans un monde de plus en plus virtuel et de plus en plus centré sur le sujet et son activité, il fallait sans doute oser ce numéro pour questionner l'usage multiple de la notion de posture dans le champ pratique et scientifique que nous connaissons actuellement. Ce numéro a pu préciser quelques définitions et situer la notion dans un champ conceptuel plus large en le mettant en relation avec des notions en proximité et en relation avec la théorie de l'activité. Par les illustrations de son usage en différents contextes (la responsabilité d'éducation de l'enseignant-chercheur à l'égard d'un public en recherche de lui-même, l'appropriation du nouveau métier de conseiller en évolution professionnelle, l'exercice de l'encadrement de futurs enseignants, le rôle d'enseignant-tuteur à l'université, etc.), il a pointé la nécessité de poursuivre l'approfondissement de cette notion. D'horizon différent, tous les cas évoqués ont chacun à leur façon, laissé entre-apercevoir le potentiel que recèle une exploration plus fine de la notion de posture professionnelle en lien avec l'activité. Un approfondissement de notre réflexion scientifique et praxéologique à son égard permettrait d'établir des ponts entre deux versants que l'enseignement supérieur a peu l'habitude de mobiliser ensemble: une dimension académique et une dimension humaine qui prend en compte l'expérience et la sensibilité du sujet. Force est de constater que les articles qui constituent ce numéro spécial posent de nombreuses questions tant au chercheur qu'au praticien et qu'il appelle donc une suite. C'est là une invitation à adresser à la revue ou à celle qui a coordonné ce numéro toute forme de réaction, de commentaire et de proposition d'articles pour continuer à approfondir cette notion omniprésente dans nos pratiques.

\section{BIBLIOGRAPHIE}

Albero, B. \& Guérin, J. (2014). Note de synthèse : L'intérêt pour l' « activité » en sciences de l'éducation : vers une épistémologie fédératrice ? TransFormations, (11), 11-45.

Allport, G.W. (1954). The historical background of modern social psychology. Dans G. Lindzey (éd.), Handbook of social psychology (Vol. 1, p. 3-56). Cambridge, MA : Addison- Wesley.

Attitude. (2010). Dans Le nouveau petit Robert. Dictionnaire alphabétique et analogique de la langue française. Paris : Dictionnaires Le Robert.

Bourdieu, P. (1987). Choses dites. Paris : Les éditions de minuit.

Carré, P. (2005). L'apprenance, vers un nouveau rapport au savoir. Paris : Dunod.

Clot, Y. (2007). De l'analyse des pratiques au développement des métiers. Education \& Didactique, (1), 83-94. 
Lameul, G. (2006). Former des enseignants à distance? Etude des effets de la médiatisation de la relation pédagogique sur la construction des postures professionnelles. Thèse de doctorat inédite, Université Paris Ouest La Défense, Paris.

Lameul, G. (2008). Les effets de l'usage des technologies d'information et de communication en formation d'enseignants sur la construction des postures professionnelles, Savoirs, (17), 73-94.

Lameul, G. \& Loisy, C. (dir.) (2014). La pédagogie universitaire à l'heure du numérique : questionnements et éclairages de la recherche. Bruxelles : éditions De Boeck.

Linard, M. (1989). Des machines et des hommes. Apprendre avec les nouvelles technologies. Paris : L'Harmattan.

Linard, M. (2002). Conception de dispositifs et changement de paradigme en formation. Education permanente, (152), 143-155.

Mulin, T. (2014). Posture professionnelle. Dans A. Jorro, Dictionnaire des concepts de la professionnalisation (p. 213-217). Bruxelles : De Boeck.

Posture. (2010). Dans Le nouveau petit Robert. Dictionnaire alphabétique et analogique de la langue française. Paris : Dictionnaires Le Robert.

Trocmé-Fabre H. (1999). Réinventer le métier d'apprendre. Paris : Edition d'Organisation.

Vallerand, R.J. (dir.) (1994). Les fondements de la psychologie sociale. Québec : Gaëtan Morin.

\section{NOTES}

1. Projet de recherche européen Dispositifs hybrides: nouvelle perspective pour une pédagogie de l'enseignement supérieur, http://prac-hysup.univ-lyon1.fr

\section{AUTEUR}

\section{GENEVIÈVE LAMEUL}

Université Bretagne Loire - Université Rennes 2 (CREAD, EA 3875), Rennes

genevieve.lameul@univ-rennes2.fr 